\section{Adherencia a tratamiento en la epilepsia: una cuestión por resolver}

\section{Adherence to treatment in epilepsy: Still an unresolved issue}

\section{Sr. Editor:}

La epilepsia es una enfermedad crónica, que afecta actualmente a más de 50 millones de personas en el mundo, $80 \%$ de las cuales vive en países en vías de desarrollo y su mayoría no es tratada de manera óptima. Según datos de la Organización Mundial de la Salud (OMS), da cuenta de 1\% de la carga global de enfermedad ${ }^{1}$. Su curva de distribución es bimodal, con una mayor prevalencia en los menores de 20 años y en los mayores de 60 años. Como ha sido documentado, la epilepsia tiene elevados costos directos (anticonvulsivantes, control médico especializado etc.) e indirectos, que sólo en Estados Unidos de Norteamérica alcanzarían a los US\$ 12.5 billones anuales ${ }^{2}$. Añádase a esto su persistente estigma e impacto sobre la calidad de vida. Como señaló Kale, "la historia de la epilepsia puede resumirse en 4.000 años de ignorancia, superstición y estigma, seguida por 100 años de conocimiento, superstición y estigma"

Pese a los avances en investigación básica y clínica, especialmente de los mecanismos epileptogénicos y los mecanismos de daño cerebral conducentes a epilepsias post traumáticas ${ }^{4}$, persiste un elevado porcentaje de pacientes sin tratar o que reciben un tratamiento inadecuado. Este porcentaje, conocido como Brecha de Tratamiento (Treatment Gap, TG), fluctúa entre 60 y $90 \%$ en los países en desarrollo, alcanzando el $58 \%$ promedio en Latinoamérica. El TG es influido por variables atribuibles al sistema de salud -como la disponibilidad de AEDs, disponibilidad de epileptólogos etc.- y variables individuales, como el sistema individual de creencias, el estigma asociado, las representaciones de la enfermedad, la adherencia, conflictos psicológicos etc. ${ }^{5}$.

De estas variables, la adherencia al tratamiento constituye por sí sola un significativo problema de salud pública, transversal a las enfermedades agudas y crónicas, aunque es más importante en las crónicas, en las que el paciente debe constituirse en agente de su propio cuidado. Definida por la OMS como "el grado en que el comportamiento de una persona se corresponde con las recomendaciones acordadas con un prestador de asistencia sanitaria”, la adherencia a tratamiento médico fluctúa en la epilepsia no refractaria entre 40 y $60 \%$ de los pacientes. Las cifras en la epilepsia refractaria son mayores, pues allí se construye desde terceros, actuando la familia y pareja básicamente como locus de control externo.

En Chile, la epilepsia es un importante problema de salud y existen sólo estimaciones del TG. Es una enfermedad cubierta por el plan estatal de salud "AUGE", tiene una prevalencia de 17 a 10,8 por cada 1.000 habitantes y una incidencia de 114 por 100.000 habitantes por año, i.e. aproximadamente 17.500 nuevos pacientes por año ${ }^{6}$. La comparación con las cifras europeas, p. ej. 0,5\% de prevalencia y $0,4 \%$ de incidencia en Alemania, revela el rol de los factores socioeconómicos en su aparición, cuidado y prevención.

Estas cifras destacan la importancia del tema, pues los pacientes no adherentes o parcialmente adherentes son más vulnerables psicopatológicamente, presentan más problemas psicosociales y psicológicos, requieren más recursos médicos, sociales y económicos y suelen presentar un curso más tortuoso y refractario de la enfermedad, con el consiguiente detrimento de su calidad de vida. Estos antecedentes justifican evaluar los factores psicológicos, psicosociales e institucionales que influyen en la conducta adherente. Ello permitiría, por una parte, predecir grupos de pacientes no adherentes, detectarlos precozmente e iniciar medidas de refuerzo. Estimularía, además, un cambio de énfasis en la aplicación de protocolos clínicos y políticas de salud, comprendiendo la conducta adherente no sólo como una variable del outcome, sino también del proceso terapéutico. En este sentido, la adherencia sería una variable dinámica e interpersonal, presente durante todo el proceso de tratamiento y que se configuraría en la interfase clínico/paciente. Este dato no es menor, pues la investigación disponible muestra que pacientes y clínicos difieren significativamente en la importancia que atribuyen a diferentes variables, p. ej. sólo $23,7 \%$ de los clínicos considera que la carga social de la epilepsia es un problema importante, en tanto que $79,4 \%$ de los pacientes lo considera un problema mayor. Curiosamente, la baja adherencia y falta de profesionales especializados en epilepsia, son percibidos cómo problema más por los clínicos que por los pacientes (40\% versus $11 \%$ para este último). Estos datos sugieren 
que un primer paso en un programa de abordaje de la adherencia en epilepsia, sería promover un acercamiento conceptual y comprensivo entre clínicos y pacientes, aunar criterios y establecer consensos en torno a los temas de mayor discrepancia, como la carga social y el estigma. Este es un camino de doble vía, en tanto implica una tarea psicoeducativa del clínico, respecto de la sociedad y de sus pacientes, pero también acoger e integrar la comprensión que tiene el paciente de su enfermar, no sólo como un énfasis en la praxis de una medicina más "humanitaria". Se trata en realidad de un problema más complejo, que implica un modelo diferente de comprensión de la enfermedad, tratamiento e indicación. Por razones de espacio no podemos extendernos en ello, pero baste señalar que comenzó su desarrollo en los Países Bajos e Inglaterra, en diferentes colectivos (Trastornos del Ânimo, Cáncer, Asma) planteando estrategias de tratamiento concordadas entre médico y paciente ("Shared decision making") dentro de ciertos límites. Los tratamientos así diseñados muestran mejoras del outcome (mayor adherencia, mejor tasa de sobrevida, disminución de los síntomas etc.).

Esta comprensión de la subjetividad del paciente y construcción de consensos, podrían fortalecer el rol del clínico y mejorar la adherencia y calidad de vida. Respecto de ésta última, los datos disponibles muestran que los factores específicos de la enfermedad, p.ej. tipo de crisis, tienen menor poder explicativo respecto de la calidad de vida y la adherencia, que los factores intrapsíquicos, como las representaciones internas de la enfermedad, los mecanismos de coping utilizados, etc. Esto refuerza el planteamiento desarrollado en esta carta.

Luis Alvarado ${ }^{1,2}$, Fernando Ivanovic-Zuvic $R_{.}^{2,3}$ ${ }^{1}$ Departamento de Psiquiatría Oriente, Facultad de Medicina. ${ }^{2}$ Departamento de Psicología, Facultad de Ciencias Sociales. ${ }^{3}$ Clínica Psiquiátrica Universitaria, División Ciencias Médicas Norte. Facultad de Medicina, Universidad de Chile. Santiago, Chile.

\section{Referencias}

1. Adherence to Long-Term Therapies. Evidence for Action. World Health Organization (2003): 3-5. Genève, Switzerland.

2. Davis KL, Candrilli SD, Edin HM. Prevalence and cost of nonadherence with antiepileptic drugs in an adult managed care population. Epilepsia 2007; 49 (3): 446-54.

3. Kale R. Bringing epilepsy out of the shadows. BMJ 1997; 315: 2-3. Disponible en: http://www.epilepsyfoundation. org/advocacy/care/genedisc.cfm (consultado el 5 de mayo de 2011).

4. ILAE. Research Priorities in Epilepsy for the next decade-A representative view of the European Scientific Community, 17-18 January 2008. Disponible en: http:// www.ilaeepilepsy.org/visitors/Documents/CEAResearchPrioritiesPositionPaper.pdf (consultado el 10 de mayo de 2011).

5. World Health Organization, Program for Neurological Diseases and Neurosciences. Department of Mental Health and Substance Abuse. Atlas-Epilepsy Care in the World. WHO 2005: 8-9. Genève, Switzerland.

6. http://www.redsalud.gov.cl/archivos/guiasges/2008/ GPC-Epilepsia2008.pdf. 